\title{
Bounds for the Radio Number of Mesh Derived Architecture
}

\author{
Kins Yenoke ${ }^{1}$, P. Selvagopal ${ }^{2}$, K.M Baby Smitha ${ }^{3}$, Ronald Cranston ${ }^{4}$ \\ Assistant Professor, Department of Mathematics, Loyola College, Chennai, India ${ }^{1}$ \\ Lecturer, Mathematics Section, Al Musanna College of Technology, Sultanate of Oman ${ }^{2}$ \\ Assistant Professor, Department of Mathematics, Jeppiaar Engineering College, Chennai, India ${ }^{3}$ \\ M.Phil Scholar, Department of Mathematics, Loyola College, Chennai, India ${ }^{4}$
}

\begin{abstract}
The radio number problem uses a graph-theoretical model to simulate optimal frequency assignments on wireless networks. A radio labelling of a connected graph $G$ is an injection $f$ from the vertices of $G$ to the natural numbers such that $d(u, w)+|f(u)-f(w)| \geq 1+\operatorname{diam}(G)$ for every pair of vertices $u$ and $w$ of $G$. The radio number of $\boldsymbol{f}$ denoted by $r n(f)$, is the maximum number assigned to any vertex of $G$. The radio number of $G$, denoted $r n(G)$, is the minimum value of $r n(f)$, taken over all labellings $f$ of $G$. In this paper we have determined the bounds for the radio number of anEnhanced mesh. These results have implications in the optimization of radio frequency assignment in wireless networks.
\end{abstract}

KEYWORDS: Labelling, Radio labelling, Radio number, Enhanced mesh.

\section{INTRODUCTION}

Radio labeling is a labeling problem which has been studied extensively in the past two decades with many different approaches, including graph theory and combinatorics, simulated annealing, genetic algorithms, and neural networks. The general problem that inspired radio labelling is what has been known as the channel assignment problem. The goal here is to assign radio channels in such a way so as to avoid interference between radio transmitters that are geometrically close. The use of graph theory to study the Channel Assignment Problem and related problems dates back at least to 1970. The problem was first put into a graph theoretic context by Hale [5] in 1980. Since then, a number of graph colourings have been inspired by the Channel Assignment Problem.

For a connected graph $G$ of diameter $d$ and an integer $k$ with $1 \leq k \leq d$; a $k$-radio colouring (sometimes called a radio $\mathrm{k}$-colouring) of $\mathrm{G}$ is an assignment $\mathrm{f}$ of colours (positive integers) to the vertices of $\mathrm{G}$ such that $d(u, w)+$ $|f(u)-f(w)| \geq 1+k$ for every two distinct vertices $u$ and $w$ of $\mathrm{G}$. When $k=d$, the $k$-radio colouring becomes a radio labelling problem which is used to assign the maximum number of channels by using minimum bandwidth. A radio labelling of a connected graph $G$ is an injection $f$ from the vertices of $G$ to the natural numbers such that $d(u, w)+|f(u)-f(w)| \geq 1+\operatorname{diam}(G)$ for every pair of vertices $u$ and $w$ of $G$. The radio number of $f$ denoted by $r n(f)$, is the maximum number assigned to any vertex of $G$. The radio number of $G$, denoted $r n(G)$, is the minimum value of $r n(f)$, taken over all labellings $f$ of $G$. The radio numbers for different families of graphs and networks were studied by several researchers. Finding the lower bound for the radio labelling is a very difficult task. Using the concept of eccentricities, Bharati et.al. [2] in 2011, proved the lower bounds for the radio number of any simple connected graph. S. K. Vaidya et.al. [9] obtained the radio number of total path of graphs.DevsiBantva[4] determined the radio number for middle graph of paths. Der-Fen Liu [3] investigated the radio number for trees. Kins Yenoke [7] obtained the upper and lower bounds for the radio number of extended mesh $\operatorname{EX}(n, n)$.

In this paper we have determined the upper and lower bounds for the radio number of the mesh derived architecture, namely the enhanced mesh.

\section{ENHANCED MESH AND ITS ECCENTRICITIES}

In this section we have given the definition of Mesh and its derived architectures. Also, the different eccentricities of enhanced mesh were calculated.

Mesh networks are highly interconnected network of computers or communication devices.Mesh networks are a way of structuring peer nodes (vertices) in such a way that each node (vertex) act as a switch or a router deciding how 


\section{\|Volume 9, Issue 6, June 2020\|}

to forward the information they receive. These networks use every node (vertex) to determine the path of the signal in communication technology.

Definition 3.1: The $\boldsymbol{m} \times \boldsymbol{n} \boldsymbol{m e s h}$ denoted $M(m, n)$ is defined as the Cartesian product $P_{m} \times P_{n}$ of paths on $m$ and $n$ vertices respectively. The number of vertices in $M(m, n)$ is $m n$ and the diameter is $m+n-2$.

Definition 3.2: The architecture obtained by making each 4-cycle in $M(m, n)$ into a complete graph is called an extended mesh. It is denoted by $E X(m, n)$.

Definition 3.3: We place a vertex in each bounded face of a $m \times n$ mesh and join it to the vertices of the face, we obtain a derived architecture called Enhanced Mesh. It is denoted by $E N(m, n)$. The number of vertices in the enhanced mesh $M(m, n)$ ismn $+(m-1)(n-1)$ and the diameter is $m+n-2$.

In this paper we name the vertices of $E N(m, n)$ as $v_{i j}$ and $u_{i j}$, where $i=1,2 \ldots m, j=1,2 \ldots n$. SeeFig.1.

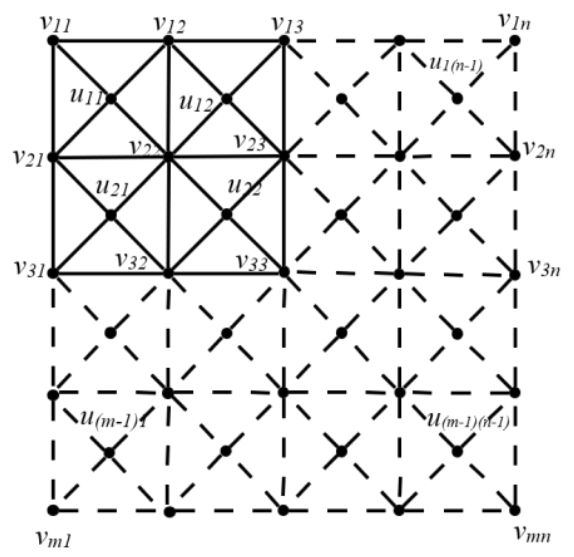

Fig.1: A general naming of vertices in $E N(m, n)$

Definition 3.4: Let $G$ be a connected graph and let $v$ be a vertex of $G$. The eccentricity $\boldsymbol{e}(\boldsymbol{v})$ of $v$ is the farthest vertex from $v$. Thus $e(v)=\max \{d(u, v) \forall u \in V(G)$.

Definition 3.5: The diameter of $\boldsymbol{G}$ is the maximum eccentricity of the vertices of $G$. It is denoted by $\operatorname{diam}(G)$.

Definition 3.6: The radius of $\boldsymbol{G}$ is the minimum eccentricity of the vertices of $G$. It is denoted by $\operatorname{rad}(G)$.

Remark 1: The eccentricities $e_{0}, e_{1} \ldots e_{n-1}$ of $E N(n, n)$ are given by $\operatorname{diam}(\operatorname{EN}(n, n))=e_{0}=2 n-2, e_{1}=2 n-3, e_{2}=$ $2 n-4, e_{3}=2 n-5 \ldots e_{\left\lceil\frac{n}{2}\right\rceil_{-1}}=n-1=\operatorname{rad}\left(E N(n, n)\right.$. That is $e_{i}=2 n-(i+2), 0 \leq i \leq\left\lceil\frac{n}{2}\right\rceil-1$.

The number of vertices having eccentricities $e_{i}$ is $8(i+1)-4, i=0,1 \ldots\left\lfloor\frac{n}{2}\right\rfloor-1$ and $e_{n-i}=8(i-1)+\left\lceil\frac{1}{i}\right\rfloor, i=0,1 \ldots\left\lceil\frac{n}{2}\right\rfloor$. See Fig.2.

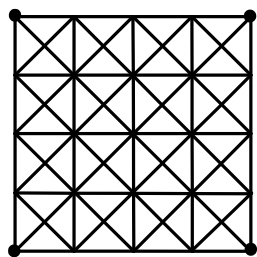

$\left(a_{1}\right)$

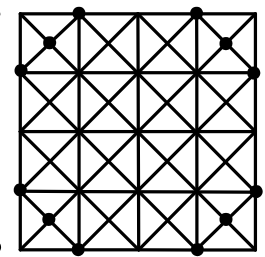

$\left(a_{2}\right)$

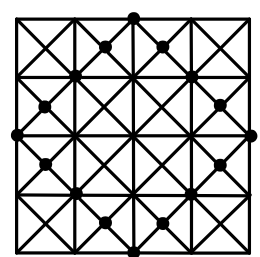

$\left(a_{3}\right)$

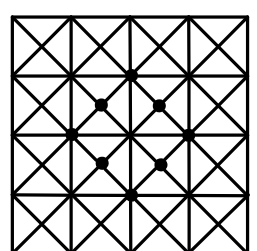

$\left(a_{4}\right)$

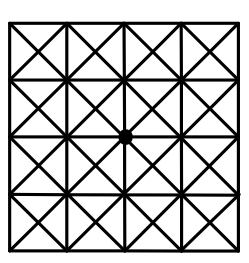

$\left(a_{5}\right)$

Fig.2: The eccentricities $e_{i}, 0 \leq i \leq 4$, for different vertices in $\mathrm{EN}(5,5)$ and are highlighted in the figures $\left(a_{i,}\right), 1 \leq i \leq 5$. 


\section{\|Volume 9, Issue 6, June 2020\|}

\section{UPPER BOUND FOR ENHANCED MESH}

In this section we determined the upper bound for the radio number of enhanced mesh $E N(n, n)$.

Theorem 3.1: Let $G$ be an enhanced mesh $E N(n, n), n \geq 4$. Then the radio number of $G$ satisfies

$$
r_{n}(G) \leq(n-2)\left(4 n^{2}-9 n+8\right)+2(n-1)^{2}+(n+1) \text {. }
$$

Proof:First we partition the vertex set $V(E N(n, n))$ into 3 disjoint sets $V_{1}, V_{2}$ and $V_{3}$. Let $V_{1}=\left\{v_{11}, v_{12} \ldots v_{(n-1) n}\right\} V_{2}=$ $\left\{u_{11}, u_{12} \ldots u_{1, n-1}, u_{21} \ldots u_{(n-1)(n-1)}\right\}$ and $V_{3}=\left\{v_{n 1}, v_{n 2} \ldots v_{n n}\right\}$.

Define a mapping $f: \operatorname{EN}(n, n) \rightarrow N$ as follows:

$f\left(v_{i j}\right)=(2 n-2)(j-1)+\left(4 n^{2}-9 n+8\right)(i-1)+1, i=1,2 \ldots n ., j=1,2 \ldots n-1$,

$$
f\left(u_{i j}\right)=(2 n-2)(n-1)+\left(4 n^{2}-9 n+8\right)(i-1)+1+n+(2 n-3)(j-1) \forall i, j=1,2 \ldots n-1,
$$

$f\left(v_{n j}\right)=(3 n-2)+(\mathrm{j}-1)(2 \mathrm{n}-2), i=1,2 \ldots n-1$ and $f\left(v_{n n}\right)=2$.

Next we show $f$ is a valid radio labelling by verifying the radio labelling condition, $d(u, w)+|f(u)-f(w)| \geq 1+$ $\operatorname{diam}(G)=1+2 n-2=2 n-1$, holds for all pair of verticesu, $w \in V(E N(n, n))$.

Case 1: If $u, w \in V_{1}$ then $u=v_{s t}, w=v_{k l}, 1 \leq s, k \leq n-1$ and $1 \leq t, l \leq n$. Therefore $d(u, w) \geq 1$ and $f(u)=$ $(2 n-2)(t-1)+\left(4 n^{2}-9 n+8\right)(s-1)+1, f(w)=(2 n-2)(l-1)+\left(4 n^{2}-9 n+8\right)(k-1)+1$.

Since both $u$ and $w$ are distinct vertices, either $s \neq k$ or $t \neq l$ or both.
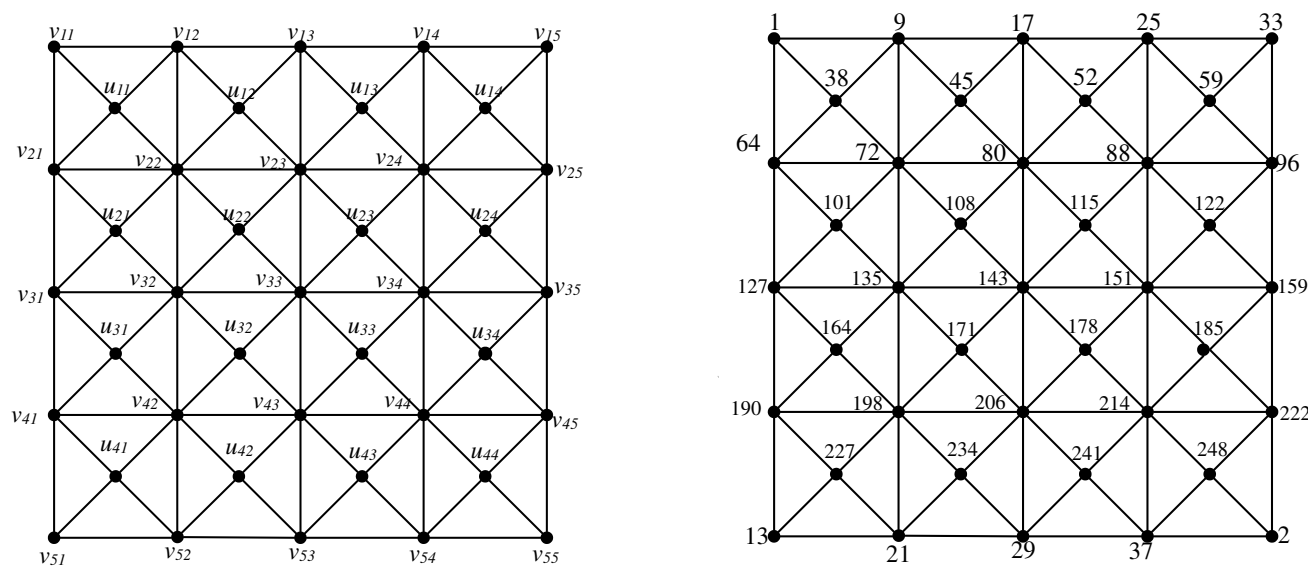

Fig. 3: The enhanced mesh $E N(5,5)$ and a radio labelling which attains the upper bound.

Subcase 1.1: If $s=$ tandt $\neq l$, then $d(u, w)+|f(u)-f(w)| \geq 1+|(2 n-2)(t-l)|=2 n-1$.

Subcase 1.2: If $s \neq$ tandt $=l$, then $d(u, w)+|f(u)-f(w)| \geq 1+\left|4 n^{2}-9 n+8\right|>2 n-1$.

Subcase 1.3: If $s \neq$ tandt $\neq l$ then $d(u, w)+|f(u)-f(w)| \geq 1+\left|(2 n-2)(t-l)+4 n^{2}-9 n+8\right|>2 n-1$.

Case 2: Suppose $v$ and ware vertices of $V_{2}$ where $u$ andware distinct, then $v=u_{l k}, w=u_{s t} 1 \leq l, s, t, n-1$.

Therefore $d(u, w) \geq 1$ and $f(v)=(2 n-2)(n-1)+\left(4 n^{2}-9 n+8\right)(l-1)+(n+1)+(2 n-3)(k-1)$, $f(w)=(2 n-2)(n-1)+\left(4 n^{2}-9 n+8\right)(s-1)+(n+1)+(2 n-3)(t-1)$.

Subcase 2.1: If $s=$ tandt $\neq l$, then $d(u, w)+|f(u)-f(w)| \geq 1+|(2 n-3)(k-t)|=2 n-1$.

Subcase 2.2: If $s \neq$ tandt $=l$, then $d(u, w)+|f(u)-f(w)| \geq 1+\left|\left(4 n^{2}-9 n+8\right)(l-s)\right|>2 n-1$.

Subcase 2.3: If $s \neq$ tandt $\neq l$ then $d(u, w)+|f(u)-f(w)| \geq 1+\left|(2 n-3)(k-t)+(l-s)\left(4 n^{2}-9 n+8\right)\right|>$ $2 n-1$.

Case 3: Suppose $u$ and $w$ are in $V_{3}$ then $u=v_{n l}, v=v_{n k}, 1 \leq l, k \leq n-1$, then $f(u)=(3 n-2)+(2 n-2)(l-1)$ and $f(w)=(3 n-2)+(2 n-2)(k-1)$. Therefored $(u, w)+|f(u)-f(w)| \geq 1|(2 n-2)(l-k)|>2 n-1$. 
Case 4: Suppose $u \in V_{1}$ and $w \in V_{2}$, then $v=u_{l k}, w=u_{s t} 1 \leq l, s, t, \leq n-1$.Also $f(u)=(2 n-2)(k-1)+$ $\left(4 n^{2}-9 n+8\right)(l-1)+1$ and $f(w)=(2 n-2)(n-1)+\left(4 n^{2}-9 n+8\right)(s-1)+(n+1)(2 n-3)(t-$ 1). Hence, $d(u, w)+|f(u)-f(w)| \geq 1+\left|(2 n-2)(k-n)+\left(4 n^{2}-9 n+8\right)(s-l)+n+(2 n-3)(t-1)\right|$.

Subcase 4.1: If $s=$ land $t \neq k$, then $d(u, w)+|f(u)-f(w)| \geq 1+\mid(2 n-2)(k-n)+\left(4 n^{2}-9 n+8\right)(0)+n+$ $(2 n-3)(t-1) \mid \geq 2 n-1$.

Subcase 4.2: If $s \neq l$ land $t=k$, then $d(u, w)+|f(u)-f(w)| \geq 1+\mid(2 n-2)(k-n)+\left(4 n^{2}-9 n+8\right)(s-l)+$ $n+(2 n-3)(k-1) \mid \geq 2 n-1$.

Subcase 4.3:If $s \neq$ tandt $\neq l$ then $d(u, w)+|f(u)-f(w)| \geq 1+\left|(2 n-3)(t-1)+(l-s)\left(4 n^{2}-9 n+8\right)\right|>$ $2 n-1$.

Case 5: Suppose $u \in V_{1}$ and $w \in V_{3}$, then $v=u_{l k}, w=u_{s t} 1 \leq l, s, t, \leq n-1 . f(u)=(2 n-2)(k-1)+$ $\left(4 n^{2}-9 n+8\right)(l-1)+1, f(w)=(3 n-2)+(2 n-2)(t-1)$ and $d(u, w) \geq 1$. Therefore $d(u, w)+$ $|f(u)-f(w)| \geq 1+\left|(2 n-2)(k-t)+(l-1)\left(4 n^{2}-9 n+8\right)-1\right|>2 n-1$.

Subcase 5.1: If $t \neq k$, then $d(u, w)+|f(u)-f(w)| \geq 1+\left|(2 n-2)(k-t)+\left(4 n^{2}-9 n+8\right)(l-1)-1\right| \geq 2 n-$ 1.

Sub case 5.2: If $t=k$, then $d(u, w)+|f(u)-f(w)| \geq 1+\left|(2 n-2)(0)+\left(4 n^{2}-9 n+8\right)(l-1)-1\right| \geq 2 n-1$.

Case 6:Suppose $u \in V_{2}$ and $w \in V_{3}$, then $u=u_{l k}, w=v_{s t} 1 \leq l, s, t, \leq n-1$. In this case $d(u, w) \geq 1, f(w)=$ $(2 n-2)(n-1)+\left(4 n^{2}-9 n+8\right)(k-1)+(n+1)+(2 n-3)(l-1)$ and $f(w)=(3 n-2)+(2 n-2)(t-1)$. Hence $d(u, w)+|f(u)-f(w)| \geq 1+\mid(2 n-2)(n-t)+(k-1)\left(4 n^{2}-9 n+8\right)+(n+1)+(2 n-3)(l-1)-$ $(3 n-2) \mid>2 n-1$.

Sub case 6.1:If $t=n$ and $l \neq k \neq 1$ then $d(u, w)+|f(u)-f(w)| \geq 1+\mid(2 n-2)(0)+(k-1)\left(4 n^{2}-9 n+\right.$ $8)+(n+1)+(2 n-3)(l-1)-(3 n-2) \mid \geq 2 n-1$.

Sub case 6.2: If $t \neq n$ and $\neq 1, k=1$, then $\quad d(u, w)+|f(u)-f(w)| \geq 1+\mid(2 n-2)(n-t)+(0)\left(4 n^{2}-9 n+\right.$ $8)+(n+1)+(2 n-3)(l-1)-(3 n-2) \mid \geq 2 n-1$.

Sub case 6.3:If $t \neq n$ and $k \neq 1, l=1$, then $d(u, w)+|f(u)-f(w)| \geq 1+\mid(2 n-2)(n-t)+(k-1)\left(4 n^{2}-\right.$ $9 n+8)+(n+1)+(2 n-3)(0)-(3 n-2) \mid \geq 2 n-1$.

Thus $d(u, w)+|f(u)-f(w)| \geq 2 n-1$ for all $u, w \in V(E N(n, n)), n \geq 4$. Since the vertex $v_{(n-1)(n-1)}$ receives the maximum label, the radio number of the enhanced $(E N(n, n))$ satisfies, $r_{n}(G) \leq(n-2)\left(4 n^{2}-9 n+8\right)+$ $2(n-1)^{2}+(n+1), n \geq 4$.

\section{LOWER BOUND FOR ENHANCED MESH}

In [2], Bharati Rajan et.al. proved the following result which we are using to find the lower bound of enhanced $\operatorname{mesh} E N(n, n)$.

Theorem 4.1: (As Theorem 2 in [2]): Let $\mathrm{G}$ be a simple connected graph of order $n$. Let $n_{0}, n_{1} \ldots n_{k}$ be the number of vertices having eccentricities $e_{0}, e_{1} \ldots e_{k}$, wherediam $(G)=e_{0}>e_{1}>\cdots>e_{k}=\operatorname{rad}(G)$.Then

$$
r_{n}(G) \geq\left\{\begin{array}{ll}
n-2\left(d-e_{k}\right)+\sum_{i=1}^{k} 2\left(d-e_{i}\right) n_{i}, & \text { if } n_{k}>1 \\
n-\left(d-e_{k}\right)-\left(d-e_{k-1}\right)+\sum_{i=1}^{k} 2\left(d-e_{i}\right) n_{i}, & \text { if } n_{k}=1
\end{array} .\right.
$$

Theorem 4.2: Let $e_{0}, e_{1} \ldots e_{n-1}$ be the eccentricities of the vertices of the enhanced mesh $E N(n, n)$ with $d=e_{0}>$ $e_{1}>\cdots>e_{n-1}=\rho . \operatorname{Then} r_{n}(G) \geq 3-n+\sum_{i=0}^{\left\lfloor\frac{n}{2}\right\rfloor-1} 2 i(8 i+4)+\sum_{i=0}^{\left[\frac{n}{2}\right\rfloor} 2 i(8 i-8)+\left[\frac{1}{i}\right]$. 
Proof: From Remark 1, we have $e_{i}=2 n-(i+2), 0 \leq i \leq\left\lceil\frac{n}{2}\right\rceil-1$ and the number of vertices having eccentricities $e_{i}$ 's are $8(i+1)-4, i=0,1 \ldots\left\lfloor\frac{n}{2}\right\rfloor-1$ and $e_{n-i}=8(i-1)+\left\lceil\frac{1}{i}\right\rfloor, i=0,1 \ldots\left\lceil\frac{n}{2}\right\rceil$.

Since $n_{k}=1$, we apply second part of Theorem 4.2 , we get

$$
\begin{aligned}
r_{n}(G) \geq n-\left(d-e_{k}\right)-\left(d-e_{k-1}\right)+\sum_{i=1}^{k} 2\left(d-e_{i}\right) n_{i} \\
=n-(2 n-2-(n-1))-(2 n-2-n)+\sum_{i=1}^{k} 2\left(2 n-2-(2 n-(i+2)) n_{i}\right. \\
=3-n+\sum_{i=1}^{\left|\frac{n}{2}\right|-1} 2 i n_{i}+\sum_{i=1}^{\left[\frac{n}{2}\right]} 2 i n_{i}
\end{aligned}
$$

Therefore, $r_{n}(G) \geq 3-n+\sum_{i=0}^{\left\lfloor\frac{n}{2} \mid-1\right.} 2 i(8 i+4)+\sum_{i=0}^{\left[\frac{n}{2}\right\rceil} 2 i(8 i-8)+\left[\frac{1}{i}\right]$.

Combining Theorem 3.1 and Theorem 4.2, we have the following theorem.

Theorem 4.3: Let $E N(n, n)$ be an enhanced mesh of order $n \times n$. If $n \geq 4$, then $3-n+\sum_{i=0}^{\left\lfloor\frac{n}{2}\right\rfloor-1} 2 i(8 i+4)+\sum_{i=0}^{\left[\frac{n}{2}\right]} 2 i(8 i-8)+\left[\frac{1}{i}\right] \leq r_{n}(G) \leq(n-2)\left(4 n^{2}-9 n+8\right)+2(n-1)^{2}+(n+1)$.

\section{CONCLUSION}

In this paper we have obtained the upper and lower bounds for the radio number of enhanced mesh. The radio number problem for Silicate network, Oxide network, Rhombic honey comb etc., are under investigation.

\section{REFERENCES}

[1] Bharati Rajan, Indra Rajasingh, Jude Annie Cynthia,"Minimum metric dimension of mesh derived architectures", Proceedings of the International Conferences of Mathematics and Computer Science, Vol. 1, pp. 153-156, 2009.

[2] Bharati Rajan, Kins Yenoke, "On the radio number of hexagonal mesh", Journal of Combinatorial Mathematics and Combinatorial Computing, Vol. 79, pp. 235-244, 2011.

[3] D. Der-Fen Liu, "Radio number for trees," Discrete Mathematics, Vol. 308, pp. 1153-1164, 2008.

[4] DevsiBantva, "Radio number for middle graph of paths", Electronics Notes in Discrete Mathematics, Vol.63, pp. 93-100, 2017.

[5] W.K. Hale, "Frequency assignment: Theory and applications", Proceedings of the IEEE, Vol 68, pp. 14971514,1980

[6] R. Khennoufa, O. Togni, "The radio antipodal and radio numbers of the hypercube”, Ars Combin., Vol 1, pp. 113, 2011.

[7] Kins Yenoke, "On the Radio number of extended mesh", Journal of Computer and Mathematical Sciences, Vol 5,pp. 358-356, 2014.

[8] B.H. Metzger, "Spectrum Management Technique", Paper presented at 38th National ORSA Meeting, Detroit, MI 1970.

[9] S. K. Vaidya1, D. D. Bantva, "Radio Number for Total Graph of Paths", ISRN Combinatorics, Vol.2013, pp. 15,2013.

[10] P. Zhang, "Radio labelings of cycles", Ars combin., Vol. 65, pp. 21-32, 2002.

[11] Mehmet Ali Balci, Pınar Dündar, “Average Edge-Distance in Graphs”Selçuk J. Appl. Math., Vol. 11, pp. 63-70, 2010. 